\title{
NB-IoT Technology based on Cellular Network
}

\section{Zhenjie Wang ${ }^{1,2, a}$, Xing $\mathrm{Xu}^{1,2, \mathrm{~b}}$,Weixing Wang ${ }^{1,2}$,Jie $\mathrm{Hu}^{1,2}$, Yueju Xue $\mathrm{X}^{1,2}$}

${ }^{1}$ College of Electronic Engineering, South China Agriculture University, Guangzhou, China

${ }^{2}$ Key Laboratory of Information Acquisition and Application in Agriculture, Guangzhou Science Technology and Innovation Commission, South China Agriculture University, GuangZhou, China

ascauwzj@stu.scau.edu.cn, bxuxing@scau.edu.cn

Keywords: Narrowband Internet of Things (NB-IoT), LTE, LPWAN

\begin{abstract}
Narrowband Internet of Things has great advantage over low speed rate, which is a new cellular technology based on 3GPP Release 13 standard. NB-IoT plays an extremely important role in the era of all things interconnected as a technology of low power consumption and wide area coverage of LPWAN (Low Power Wide Area Network). This paper mainly discusses the technical superiority, standard and technical principle of NB-IoT, and proposes a set of research area based on agriculture applications.
\end{abstract}

\section{Introduction}

Now the proportion of Internet of Things technology in industry applications is growing every year, and the IoT technology has infiltrated into manufacturing, transportation and logistics, health care, consumer electronics, retail, automotive and other applications [1]. The era of all things interconnected is coming into our lives in the fast pace. One of the foundations of "all things interconnected" is that the different Internet of things have different requirements for data transmission and real-time.

There are many kinds of communication technology in Internet of things, it can be divided into two categories:

1) Short-range communication technology: Zigbee, Wi-Fi, Bluetooth, Z-wave, etc., the typical application scenarios such as smart home;

2) WAN communication technology: which is generally defined as LPWAN by industry and the typical application scenarios such as intelligent meter reading.

The current technology is mainly depend on 2G and 3G systems. The general needs of cellular networking, such as wide area coverage, indoor reception, low power consumption, low cost and support for large number of device access, can be achieved by the latest 3GPP standard NB-IoT technology based on 4G technology.

\section{NB-IoT technology advantages}

As a technology used in low-speed communication, NB-IoT has four major advantages, as follows:

1) Wide coverage. NB-IoT technology boosts the $20 \mathrm{~dB}$ gain compared to the GPRS base station. Underground garage and underground pipes can also be covered.

2) Low power consumption. NB-IoT with PSM and eDRX can achieve longer standby. Low power consumption is an important indicator of the application of things and NB-IoT terminal module's standby time can be up to 10 years.

3) Low cost. Low speed, low power consumption, low bandwidth brings low cost advantage. The current cost of a single module do not exceed $\$ 5$, and the goal is to cut prices to about $\$ 1$.

4) Large links. In the case of the same base station, NB-IoT can provide 50-100 times the number of accesses than existing wireless technologies. A sector can support 100,000 connections, supporting low latency sensitivity, low cost of equipment, low power consumption and optimized network architecture [2]. 
These advantages make NB-IoT very suitable for sensing, measurement, monitoring and other networking applications for intelligent meter reading, intelligent parking, vehicle tracking, logistics monitoring, sharing bicycles and smart wear, intelligent family, etc. [3]. 2G/3G/4G network and other wireless technologies currently used widely are unable to meet these challenges.

\section{NB-IoT key technology}

The NB-IoT standard was taken only 8 months to be frozen, and it cost the least time in the 3GPP standards. After completing the performance standards development in September 2016 and completing the conformance test in December, several major operators are currently competing to lay out the NB-IoT base station network, so it is expected that the NB-IoT will enter the mass commercial stage [4].

NB-IoT can solve the problem of indoor coverage enhancement, support huge amount of low-rate equipment access, low latency sensitive, low cost of equipment, low power consumption and network architecture optimization, mainly due to its own key technology.

\subsection{NB-IoT network deployment mode}

NB-IoT supports three deployment modes: stand-alone deployment, guard deployment, in-band deployment, as shown in Figure 1:
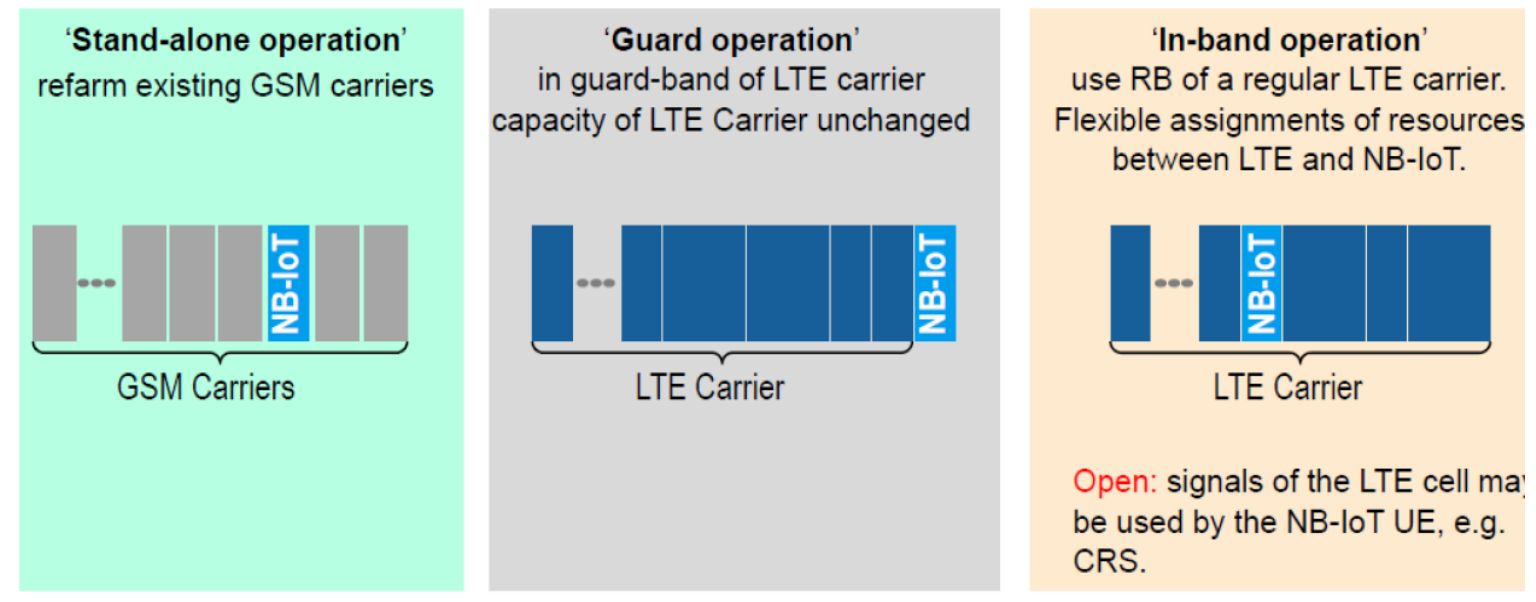

Fig.1 NB-IoT three deployment mode

1) Stand-alone (Stand-alone): can use a separate band, suitable for GSM band re-farming;

2) Guard band deployment (Guard-band): can use the edge of the LTE system useless band, bandwidth 180kHz;

3) In-band: Any resource block in the middle of the LTE carrier can be used.

With three different types of network deployments, the NB-IoT makes full and efficient use of spectrum resources.

\subsection{NB-IoT half-duplex mode}

In the 3GPP R13 version, the NB-IoT only supports the FDD half-duplex type-B mode [5]. In the type- $B$, the UE does not receive the downlink signal when the upstream signal and the subsequent subframe are transmitted, so that the guard slot is lengthened, which reduces the requirement of the device and improves the reliability of the signal. 


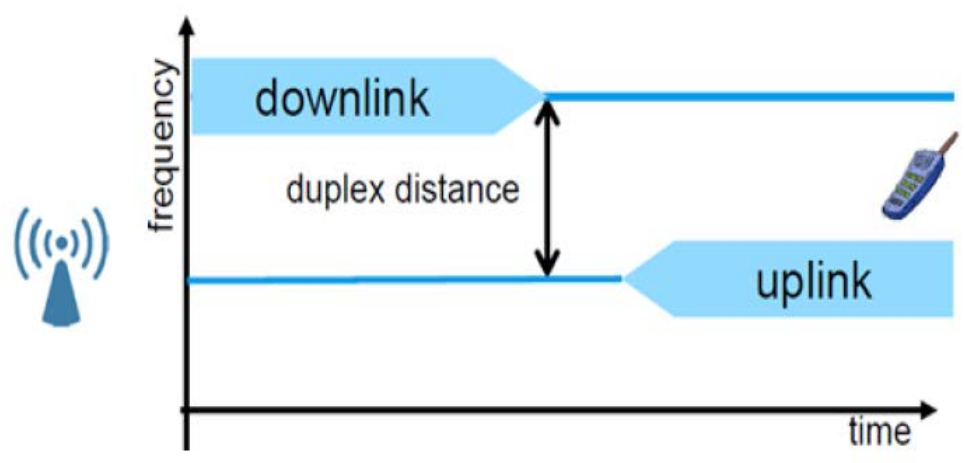

Fig.2 NB-IoT half-duplex mode of operation

\subsection{Changes to the NB-IoT physical layer}

NB-IoT system downlink transmission bandwidth of $180 \mathrm{kHz}$, using the same LTE $15 \mathrm{kHz}$ subcarrier spacing, downlink multiple access mode O for OFDMA, frame structure and physical resource units are also used to follow the existing LTE design.

The NB-IoT system reduces the type of downstream physical channel and redesigns some downstream physical channels, synchronization signals and reference signals for the $180 \mathrm{kHz}$ downlink transmission bandwidth and the need for depth coverage.

The NB-IoT system has an uplink transmission bandwidth of $180 \mathrm{kHz}$ and supports two subcarrier intervals: $3.75 \mathrm{kHz}$ and $15 \mathrm{kHz}$. For coverage enhancement scenarios, the $3.75 \mathrm{kHz}$ subcarrier spacing can provide a wider coverage than the $15 \mathrm{kHz}$ subcarrier spacing.

\subsection{NB-IoT low power technology}

NB-IoT with PSM and eDRX can achieve longer standby, as follows:

PSM (Power Saving Mode). In this mode, the terminal is still registered in the network but the signaling is unreachable, so that the terminal stay in the deep sleep for a long time to achieve the purpose of saving power.

eDRX (Enhanced Discontinuous Reception). eDRX is one of the new features of R13, which extend the non-continuous reception. It makes terminal further extend the sleep cycle in the idle mode, reducing the unnecessary start of the receiving unit, and greatly enhances the downstream reachability.
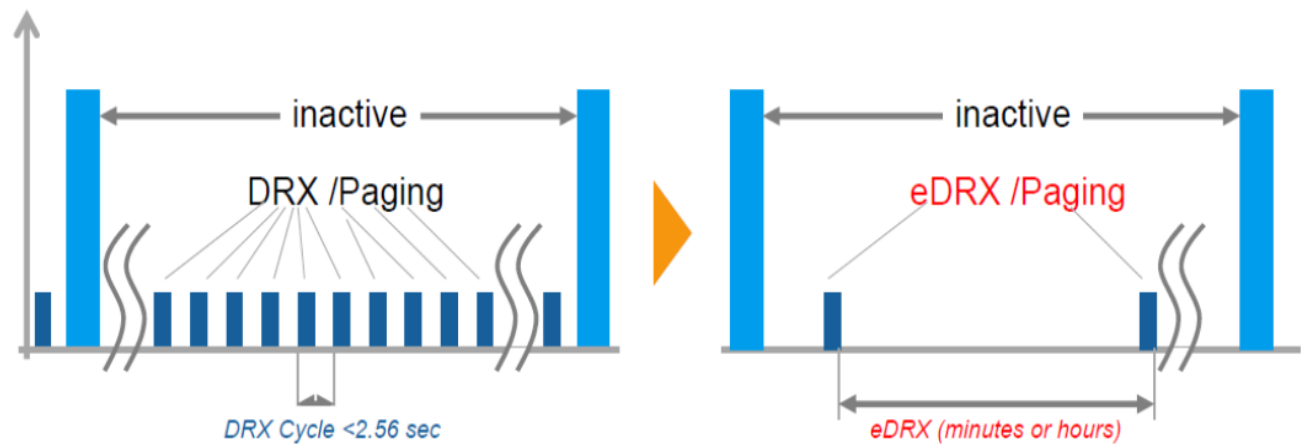

For devices with infrequently uplink data transmission, energy consumption can be reduced significantly by longer cycles
for discontinuous reception (DRX).

Fig.3 NB-IoT low power technology PSM \& eDRX

The target of NB-IoT is to build al low-rate, low-frequency business model with an equivalent battery life of more than 10 years. According to the simulation data of TR45.820, PSM and eDRX are deployed in the harsh environment with $164 \mathrm{~dB}$ of coupling loss. If the terminal sends 200byte packets every day, the battery life can reach 12.8 years. 


\section{NB-IoT Applications}

NB-IoT has great advantage in agricultural applications and industry applications compared with Zigbee and GPRS technology. It is knows that Zigbee is low power WSN technology, but it can't connect to remote server directly. GPRS module can connect to remote server, but it needs more power and is more expensive than Zigbee module. NB-IoT has the advantage of both Zigbee and GPRS, so it has great development prospect especially in agriculture area. There have three agriculture environment: field, orchard and livestock.

1) Field Environment

Field crop is usually located in plain areas and have very large planting area. So it needs large-scale monitoring. The Zigbee-based WSN network need numerous monitoring nodes and routes, and its network topology is complex. The NB-IoT will be able to greatly reduce the required routing nodes and have the advantages of stable operation. Therefore, the application of NB-IoT in the field will be the future development trend.

2) Orchard Environment

The orchard environment includes plains and mountain orchards. The mountain orchards are susceptible to interference due to terrain, so the wireless signal transmission is susceptible to interference and influence. The transmission distance between the nodes is limited due to the complex orchard environment. The collected data are difficult to send to the remote server side. The domestic communications companies invest a lot of money to achieve a nationwide coverage of mobile base stations, so the NB-IoT network application in the orchard environment has the foundation of reliable transmission, which can guarantee the mountain orchard environmental monitoring data transmission. Therefore, the orchard application of NB-IoT has obvious communication advantages.

3) Livestock Environment

In the livestock and poultry breeding area, the livestock and poultry animal object has the mobile characteristics. In this case, packet loss and network instability will occur because the WSN network is designed for static monitoring nodes. The NB-IoT technology is proposed in the mobile communication 3GPP standard, and it has the ability to support for mobile objects. So NB-IoT in livestock and poultry breeding also has a broad application prospects.

\section{Summary}

NB-IoT focuses specifically on indoor coverage, low cost, long battery life and enables a large number of devices connected. And it has a great prospect in agriculture, such as field environment, orchard environment and livestock environment. NB-IoT is ready to unlock the full potential of IoT.

\section{Acknowledgements}

This work was financially supported by Natural Science Foundation of Guangdong Province, China (2015A030310334), Science and Technology Planning Project of Guangdong Province, China (2014A020208110, 2014A020208108, 2016A020210091), Project on the Integration of Industry, Education and Research of Dongguan city (2015509130210), and Science and Technology Program of Guangzhou, China (201605030013).

\section{References}

[1] IoT at a crossroads in 2017 on http://www.fiercewireless.com/tech/

[2] What is NB-IoT on https://5g.co.uk/guides/what-is-narrowband-iot/

[3] Ratasuk, R., Mangalvedhe, N., Zhang, Y., Robert, M. Overview of narrowband IoT in LTE Rel-13. In Standards for Communications and Networking (CSCN), 2016, October, p. 1-7 
[4] Narrowband IoT (NB-IoT) RP-151621 on http://www.3gpp.org/ftp/tsg_ran/TSG_RAN/ TSGR_69/ Docs/RP-151621.zip

[5] Palattella, M.R., Dohler, M., Grieco, A., Rizzo, G., Torsner, J., Engel, T. and Ladid, L., Internet of things in the 5G era: Enablers, architecture, and business models. IEEE Journal on Selected Areas in Communications, vol.34(2016), p.510-527. 